\title{
Can diet influence cardiac geometry and function in young adults?
}

${ }^{1}$ Afifah Mohamed, MSc, ${ }^{1}$ Adam J Lewandowski, DPhil, ${ }^{1}$ Odaro Huckstep, MSc, ${ }^{1 *}$ Paul Leeson, PhD FRCP

${ }^{1}$ Oxford Cardiovascular Clinical Research Facility, Division of Cardiovascular Medicine, Radcliffe Department of Medicine, University of Oxford

*Correspondence to: Professor Paul Leeson, Oxford Cardiovascular Clinical Research Facility, University of Oxford, John Radcliffe Hospital, Oxford, OX39DU, UK.

Email: paul.leeson@cardiov.ox.ac.uk

Keywords: Salt intake, left ventricular mass index, young adults, hypertension 
High sodium intake is associated with increased blood pressure and increased future risk of stroke and heart attack, ${ }^{1}$ which has led to global initiatives in current prevention guidelines to reduce dietary sodium levels. ${ }^{2}$ How early the effects of high sodium intake are seen on the cardiovascular system has been less well studied. In this issue of the European Journal of Preventive Cardiology, Strauss et al. provide novel insights into underlying salt regulation and sensitivity via marinobufagenin (MBG) adrenal release and demonstrate that this relates to cardiac geometry and function in young adulthood. ${ }^{3}$

Their study included a total of 711 young, healthy adults from South Africa, including black, white, male and female adults with greater than $5 \mathrm{~g}$ of sodium $\left(\mathrm{Na}^{+}\right)$intake daily. Previous studies in this area were performed in animals or older human cohorts with disease progression, and thus the authors should be acknowledged for enhancing our understanding of the potential early pathophysiological implications of increased MBG excretion in a young adult population. Their main findings were that increased MBG excretion relates to $\mathrm{Na}^{+}$intake and to sub-clinical changes in left ventricular geometry and function. These changes included a higher left ventricular mass index, which remained significant after adjustment for salt intake, and 24-hour excretion. These effects were more pronounced among women, in whom sensitivity to MBG was higher despite the lower salt consumption. These findings provide further evidence that subclinical cardiac target organ damage can start to emerge during young adulthood, in response to various early life, ${ }^{4}$ environmental and pathogenic factors. ${ }^{5}$ An interesting feature of the current article is the importance of a dietary factor, high salt intake, in driving these changes and the implication of MBG in this process.

In the current study, there was not a detailed exploration of underlying mechanisms, and future studies to understand what may be inducing the increase in cardiac mass will be of interest. The increased left ventricular mass reported in those with increased MBG excretion is unlikely to be due to blood pressure elevation, as the average 24-hour blood pressure reading in this study was within the normal range. The echocardiographic findings also did not show a hypertensive pattern of left ventricular remodelling, with clinically normal values of relative wall thickness across the four quartiles of MBG excretion. In spite of this, the highest left ventricular mass was found in the fourth quartile of MBG, in which increased MBG was associated with significantly higher 24-hour urinary profile, including volume, $\mathrm{Na}+, \mathrm{K}+$ and creatinine excretion. Importantly, renal function was preserved, suggesting that a specific renal problem with salt loading is unlikely to be the primary issue for these young adults. High salt intake causes disruption of microvessels, and microvascular dysfunction is seen in early target 
organ damage associated with hypertension and other cardiovascular diseases. ${ }^{6}$ Thus, evaluation of microvascular changes and the impact on cardiac perfusion and fibrosis in these young adults may be of interest. The wider impact of these cardiac geometric changes at a young age also needs consideration. Although the authors reported no baseline differences in left ventricular function, it is now known that early changes in cardiac mass and geometry can be linked with reductions in functional myocardial reserve. Therefore, assessing how the heart responds to physiological stress using echocardiography may reveal subclinical changes in the stress response. ${ }^{7}$ Furthermore, novel cardiac imaging now offers the opportunity for detailed myocardial tissue characterization ${ }^{8}$ to define whether the increased cardiac size relates to myocardial cell hypertrophy or other factors such as fibrosis.

In conclusion, a major strength of the study is that it provides clinical evidence of how high sodium intake links with MBG excretion and cardiovascular health. Of particular note is the young age at which these cardiac changes have been identified. This suggests that subclinical target organ damage may accumulate during early adulthood in a way that may alter trajectories of disease development in response to subsequent declines in other organ systems such as renal function, vascular responses, emergence of hypertension or other cardiovascular risks. There are a limited number of cardiovascular prevention randomised trials in younger populations that have been demonstrated to provide long-term benefit. ${ }^{9}$ In view of the strong relationship between dietary salt intake and the risk of developing hypertension and future cardiovascular disease in older populations, recent examples of salt reduction and dietary trials ${ }^{10,11}$ may need to be adapted for young adult populations. Findings from such studies will then be able to define optimal strategies to maintain cardiovascular health and prevent future disease.

\section{Declaration of conflicting interests}

The authors declared no potential conflicts of interest with respect to the research, authorship and/or publication of this article.

\section{Funding}

The authors received no financial support for the research, authorship and/or publication of this article. 


\section{REFERENCES}

1. Aburto NJ, Ziolkovska A, Hooper L, et al. Effect of lower sodium intake on health: Systematic review and meta-analyses. BMJ 2013; 346: f1326.

2. Authors/Task Force M, Piepoli MF, Hoes AW, et al. 2016 European Guidelines on cardiovascular disease prevention in clinical practice: The Sixth Joint Task Force of the European Society of Cardiology and Other Societies on Cardiovascular Disease Prevention in Clinical Practice (constituted by representatives of 10 societies and by invited experts): Developed with the special contribution of the European Association for Cardiovascular Prevention \& Rehabilitation (EACPR). Eur J Prev Cardiol 2016; 23: NP1-NP96.

3. Strauss M, Smith W, Kruger R, et al. Marinobufagenin and left ventricular mass in young adults: The African-PREDICT study. Eur J Prev Cardiol 2018; 2047487318788140. 2018/07/12. DOI: 10.1177/2047487318788140.

4. Lewandowski AJ, Augustine D, Lamata P, et al. Preterm heart in adult life: Cardiovascular magnetic resonance reveals distinct differences in left ventricular mass, geometry, and function. Circulation 2013; 127: 197-206.

5. Lazdam M, Lewandowski AJ, Kylintireas I, et al. Impaired endothelial responses in apparently healthy young people associated with subclinical variation in blood pressure and cardiovascular phenotype. Am J Hypertens 2012; 25: 46-53.

6. Anderson TJ, Charbonneau F, Title LM, et al. Microvascular function predicts cardiovascular events in primary prevention: Long-term results from the Firefighters and Their Endothelium (FATE) study. Circulation 2011; 123: 163-169.

7. Huckstep OJ, Williamson W, Telles F, et al. Physiological stress elicits impaired left ventricular function in preterm-born adults. J Am Coll Cardiol 2018; 71: 1347-1356. 
8. Salerno M, Sharif B, Arheden $\mathrm{H}$, et al. Recent advances in cardiovascular magnetic resonance: Techniques and applications. Circ Cardiovasc Imaging 2017; 10.

9. Williamson W, Foster C, Reid H, et al. Will exercise advice be sufficient for treatment of young adults with prehypertension and hypertension? A systematic review and meta-analysis. Hypertension 2016; 68: 78-87.

10. Eyles H, McLean R, Neal B, et al. A salt-reduction smartphone app supports lower-salt food purchases for people with cardiovascular disease: Findings from the SaltSwitch randomised controlled trial. Eur J Prev Cardiol 2017; 24: 1435-1444.

11. Jaakkola JM, Pahkala K, Ronnemaa T, et al. Longitudinal child-oriented dietary intervention: Association with parental diet and cardio-metabolic risk factors. The Special Turku Coronary Risk Factor Intervention Project. Eur J Prev Cardiol 2017; 24: 1779-1787. 